\title{
Acoustic Emission Source Localization System Using Fiber Bragg Grating Sensors and a Barycentric Coordinate-Based Algorithm
}

\author{
Dandan Pang $\left(\mathbb{D},{ }^{1,2}\right.$ Qingmei Sui, ${ }^{3}$ Ming Wang, ${ }^{1,2}$ Yaozhang Sai, ${ }^{3}$ Rongyao Sun, ${ }^{1}$ \\ and Yikang Wang ${ }^{1}$ \\ ${ }^{1}$ School of Information and Electrical Engineering, Shandong Jianzhu University, Jinan 250101, China \\ ${ }^{2}$ Shandong Provincial Key Laboratory of Intelligent Buildings Technology, Jinan 250101, China \\ ${ }^{3}$ School of Control Science and Engineering, Shandong University, Jinan 250061, China
}

Correspondence should be addressed to Dandan Pang; pangdandan@sdjzu.edu.cn

Received 23 February 2018; Revised 6 June 2018; Accepted 11 July 2018; Published 15 August 2018

Academic Editor: Stephen James

Copyright ( 2018 Dandan Pang et al. This is an open access article distributed under the Creative Commons Attribution License, which permits unrestricted use, distribution, and reproduction in any medium, provided the original work is properly cited.

\begin{abstract}
Structures and machines are very susceptible to some barely visible defects; acoustic emission (AE) is an effective technique of detecting defects and examining growth and location of these defects. In this paper, a new method was proposed to predict the position of the AE source. The dynamic strain signal from the AE source was acquired by fiber Bragg grating (FBG) sensors. Complex Morlet wavelet transform was used to extract narrowband signals from AE waves. It was proposed that the barycentric coordinate-based localization algorithm can be used to predict the coordinates of the AE source. The validation tests based on the designed $\mathrm{AE}$ detection system were performed on a $500 \mathrm{~mm} \times 500 \mathrm{~mm} \times 2 \mathrm{~mm} \mathrm{Al}$-alloy plate. The experimental results show that the proposed method is feasible.
\end{abstract}

\section{Introduction}

The degradation of structural properties is well considered as an enormous threat for security and performance of structures and machines. Structural health monitoring (SHM) utilizes several kinds of sensors attached to or embedded in the monitored structures to detect the appearance, location, and severity of damage. Thus, SHM is imperative for damage detection and structural failure. Various methods have been researched for structural damage detection. These methods can be divided into two categories: model-based and signalbased approaches. The former develops suitable model and analyzes changes that relate to damage in the model; the latter extracts features and establishes a relationship between these features and potential damage [1-3]. Acoustic emission (AE) is one of the effective signal-based means implemented for damage detection [4].

$\mathrm{AE}$ wave is generated by energy release due to a propagating crack or friction in structures. AE technique is used to detect, locate, and assess defects. To obtain $\mathrm{AE}$ wave, some $\mathrm{AE}$ sensors have been widely used, such as piezoelectric sensors (PZT) and capacitive AE sensors [5-8]. However, the traditional AE sensors have the disadvantages such as complex structure, weak antielectromagnetic interference, and unsuitability for distributed measurement. At present, fiber Bragg grating (FBG) sensors have been expected as an alternative because of their excellent performance. FBG sensors can be easily embedded in the monitored structure without destructive effect, due to their small size and light weight [9]. Furthermore, FBG sensors are immune to electromagnetic interference [10]. In addition, long-distance distributed measurement can be realized by using FBG sensors [11]. Therefore, some scholars have done several researches on AE source localization using FBG sensors $[12,13]$. Yu et al. [14] investigated the effective length of the FBG sensor from the $\mathrm{AE}$ source on the composite plate. Yu et al. [15] proposed an AE source identification method based on the algebraic reconstruction algorithm and $3 \mathrm{D}$ imaging technique.

Recently, an increasing number of AE source localization methods was developed based on the intelligent algorithm $[16,17]$. Cheng et al. [18] applied an optimized wavelet 


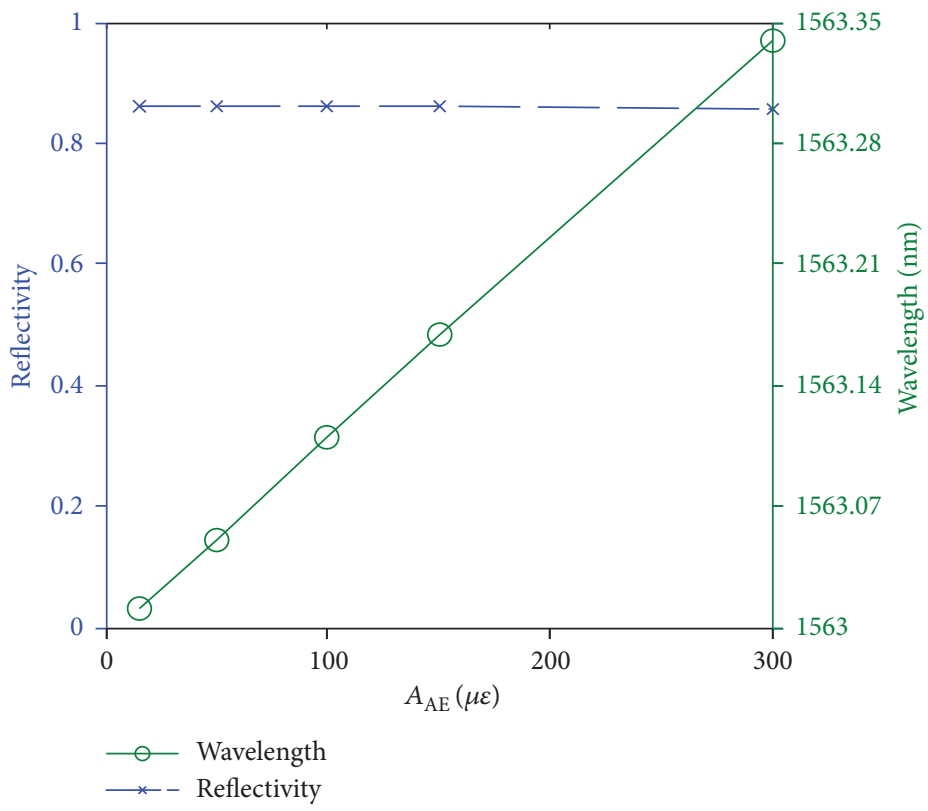

Figure 1: Relation between $A_{\mathrm{AE}}$ and reflectivity and wavelength of FBG.

neural network to realize AE source location in rotating machinery. Sadegh et al. [19] applied the genetic algorithm and artificial neural networks to extract features of AE signals for monitoring lubrication conditions of a journal bearing. However, in the intelligent algorithms used in those studies, lots of training samples were needed, which leaded to inefficiency and complexity of the localization process. Furthermore, AE source localization in plate-like structures usually requires lots of sensors. In particular, those approaches are usually accurate only within the convex area surrounded by sensors.

Based on the previous studies, this paper presented a novel AE detection system based on FBG sensors and a new AE source location method. The AE signal was collected by the FBG sensor which was pasted on the structural surface. Barycentric coordinate-based location method was used to predict positioning. Different from the approaches mentioned above, the proposed method was developed based on only range measurements between sensors, not training samples. The AE source is not required to lie inside the convex area formed by sensors. At last, AE source location experiments were carried out to verify this new designed system and localization algorithm. The results show that it is an efficient and feasible method for AE source localization.

\section{Theoretical Principle}

2.1. FBG AE Sensing Principle. The dynamic strain field along the FBG generated by $\mathrm{AE}$ wave can be simplified as a time-dependent cosine-Gauss function:

$$
\varepsilon_{\mathrm{AE}}(t)=A_{\mathrm{AE}} e^{-\left(\left(t-t_{0}\right)^{2} / a^{2}\right)} \cos \left(\frac{2 \pi}{\lambda_{\mathrm{AE}}} z-\omega_{\mathrm{AE}} t\right),
$$

where $A_{\mathrm{AE}}$ is the amplitude of $\mathrm{AE}$ wave propagating in a material to be monitored, $\lambda_{\mathrm{AE}}$ is the wavelength of $\mathrm{AE}$ wave,
$\omega_{\mathrm{AE}}$ is the angular frequency of $\mathrm{AE}$ wave, $t_{0}$ is the arrival time, and $a$ is the duration of $\mathrm{AE}$ wave.

The alternation of wavelength of FBG is mainly attributed to two factors: one is the modulation of the grating period $\Lambda$ (geometric effect), which causes the axial deformation of FBG, and the other is the change of the effective refractive index of FBG $\bar{n}_{\text {eff }}$ (photoelastic effect).

In consideration of the geometric effect and photoelastic effect, the expression of the effective refractive index of FBG under $\mathrm{AE}$ wave is obtained:

$$
\begin{aligned}
n_{\mathrm{eff}}^{\prime \prime \prime}\left(z^{\prime}, t\right)= & n_{\mathrm{eff} 0}-\Delta n \sin ^{2}\left(\frac{\pi}{\Lambda_{0}} z\right) \\
& -\frac{n_{\mathrm{eff} 0}^{3}}{2} \cdot\left[P_{12}-v\left(P_{11}+P_{12}\right)\right] \\
& \cdot A_{\mathrm{AE}} e^{\left(-\left(t-t_{0}\right)^{2} / a^{2}\right)} \cos \left(\frac{2 \pi}{\lambda_{\mathrm{AE}}} z^{\prime}-\omega_{\mathrm{AE}} t\right),
\end{aligned}
$$

where $z^{\prime}=z+\int_{0}^{z} \varepsilon(\xi) d \xi, P_{11}$ and $P_{12}$ are the photoelastic coefficients of FBG, and $v$ is Poisson's ratio [20].

Based on the mentioned theory above, the spectrum characteristics of FBG under AE waves are simulated and analyzed. When the wavelength of $\mathrm{AE}$ wave $\lambda_{\mathrm{AE}}$ is $100 \mathrm{~mm}$, the length of grating $L$ is $10 \mathrm{~mm}$ and the amplitudes of $\mathrm{AE}$ waves $A_{\mathrm{AE}}$ are $15 \mu \varepsilon, 50 \mu \varepsilon, 100 \mu \varepsilon, 150 \mu \varepsilon$, and $300 \mu \varepsilon$, respectively. Relation between $A_{\mathrm{AE}}$ and reflectivity and wavelength of FBG is shown in Figure 1. The center of FBG shifts towards the longer wavelength as $A_{\mathrm{AE}}$ increases when $\lambda_{\mathrm{AE}}$ is greater than $L$. Results also show that there is a good linear relation between central wavelength and $A_{\mathrm{AE}}$. The maximum reflectivity of the FBG reflection spectrum keeps invariant as the change of $A_{\mathrm{AE}}$.

2.2. Complex Morlet Wavelet Transform. The collected AE waves often contain some mutation-shaped peaks or 
nonstationary components. Morlet wavelet transform is employed to extract the narrowband signal of AE waves.

The continuous wavelet transform of AE wave $\varepsilon_{\mathrm{AE}}(t)$ can be defined as

$$
W_{\varepsilon}(\alpha, \tau)=\frac{1}{\sqrt{\alpha}} \int_{-\infty}^{+\infty} \varepsilon_{\mathrm{AE}}(t) \bar{\phi}\left(\frac{t-\tau}{\alpha}\right) d t
$$

where $1 / \sqrt{\alpha} \bar{\phi}(t-\tau / \alpha)$ is the complex conjugate of the scaled wavelets $1 / \sqrt{\alpha} \phi(t-\tau / \alpha)$ obtained by zooming and panning the mother wavelet, $\alpha$ is the zoom factor, and $\tau$ is the shift factor.

The complex Morlet wavelet can be expressed as [21,22]

$$
\phi(t)=\frac{1}{\sqrt{\pi f_{\mathrm{b}}}} e^{-\left(t^{2} / f_{\mathrm{b}}\right)} e^{i 2 \pi f_{\mathrm{c}} t}
$$

The Fourier transform of the above function is defined by

$$
\varphi(\omega)=e^{-\pi^{2} f_{\mathrm{b}}\left(\omega-f_{\mathrm{c}}\right)^{2}}
$$

where $f_{\mathrm{b}}$ and $f_{\mathrm{c}}$ are the bandwidth factor and center frequency of the complex Morlet wavelet, respectively. The wavelet is a complex exponential function with the shape of a Gaussian window. Hence, the narrowband signal with center frequency $f_{\mathrm{c}}$ can be extracted by Morlet wavelet transform. The bandwidth is limited in the range of $\left(\left(\omega_{c}-\omega_{b}\right) / 2,\left(\omega_{c}-\omega_{b}\right) / 2\right)$. The complex Morlet wavelet filter can be constructed as

$$
W T\left(f_{\mathrm{b}}, f_{\mathrm{c}}\right)=F^{-1}\left[E_{\mathrm{AE}}(\omega) \bar{\varphi}(\omega)\right]
$$

where $F^{-1}$ denotes the inverse Fourier transform, $E_{\mathrm{AE}}(\omega)$ and $\varphi(\omega)$ are the Fourier transform of $\varepsilon_{\mathrm{AE}}(t)$ and $\phi(t)$, respectively.

When considering the propagation distance $z$, the $\mathrm{AE}$ wave $\varepsilon_{\mathrm{AE}}(z, t)$ can be express as

$$
\varepsilon_{\mathrm{AE}}(z, t)=e^{-j\left(n_{1} z-\omega_{1} t\right)}+e^{-j\left(n_{2} z-\omega_{2} t\right)}
$$

where $n_{1}$ and $n_{2}$ are the wave numbers. Denote

$$
\begin{aligned}
& \frac{n_{1}+n_{2}}{2}=n_{0}, \\
& \frac{n_{1}-n_{2}}{2}=\Delta n, \\
& \frac{\omega_{1}+\omega_{2}}{2}=\omega_{0}, \\
& \frac{\omega_{1}-\omega_{2}}{2}=\Delta \omega, \\
& \bar{\varphi}(\alpha \omega)=\bar{\varphi}\left(\alpha \omega_{1}\right)=\bar{\varphi}\left(\alpha \omega_{2}\right) .
\end{aligned}
$$

The module value of $\mathrm{AE}$ wave is acquired by the complex Morlet wavelet transform

$$
|W T(z, \alpha, \tau)|=\sqrt{2 a}\left|\varphi\left(\alpha \omega_{0}\right)\right| \sqrt{1+\cos (2 \Delta n z-2 \Delta \omega \tau)} .
$$

When $\tau=\Delta n z / \Delta \omega=z / V$, the module value is maximum, where $V$ is the group velocity of $\mathrm{AE}$ wave. Therefore, the arrival time difference $\Delta t$ can be obtained by finding the peak values of the module values:

$$
\Delta t=\tau_{1}-\tau_{2}
$$

According to the arrival time difference and the distance difference, the wave velocity $v_{\mathrm{AE}}$ can be calculated on the monitored material:

$$
v_{\mathrm{AE}}=\frac{\Delta z}{\Delta t}
$$

2.3. Barycentric Coordinate-Based Location Algorithm. In 1827, the barycentric coordinate was developed to characterize the relative position of a point with respect to other points [23-26]. In the plane, for a point $k$ and other three points $l, m$, and $n$, the barycentric coordinate of point $k$ with respect to points $l, m$, and $n$ can be defined as $\left\{a_{k l}, a_{k m}\right.$, and $\left.a_{k n}\right\}$ that satisfies

$$
p_{k}=a_{k l} p_{l}+a_{k m} p_{m}+a_{k n} p_{n}
$$

where $p_{k}, p_{l}, p_{m}$, and $p_{n}$ are the Euclidean coordinates of points $k, l, m$, and $n$, respectively.

Consider an $\mathrm{AE}$ event $k$ occurs on the plate with three sensors $l, m$, and $n$ nearby, the AE signal is detected by each of the sensors. Then, the absolute values of the barycentric coordinates $a_{k l}, a_{k m}$, and $a_{k n}$ can be computed as

$$
\begin{aligned}
& \left|a_{k l}\right|=\frac{\left|S_{\Delta k m n}\right|}{\left|S_{\Delta l m n}\right|}, \\
& \left|a_{k m}\right|=\frac{\left|S_{\Delta k n l}\right|}{\left|S_{\Delta l m n}\right|}, \\
& \left|a_{k n}\right|=\frac{\left|S_{\Delta k l m}\right|}{\left|S_{\Delta l m n}\right|},
\end{aligned}
$$

where $S_{\Delta k m n}, S_{\Delta k n l}, S_{\Delta k l m}$, and $S_{\Delta l m n}$ are the signed areas of the corresponding triangles $\Delta k m n, \Delta k n l, \Delta k l m$, and $\Delta l m n$. These areas can be solved according to

$$
S_{\Delta k m n}^{2}=-\frac{1}{16}\left|\begin{array}{cccc}
0 & 1 & 1 & 1 \\
1 & 0 & d_{k m}^{2} & d_{k n}^{2} \\
1 & d_{m k}^{2} & 0 & d_{m n}^{2} \\
1 & d_{n k}^{2} & d_{n m}^{2} & 0
\end{array}\right|,
$$

where $d_{k m}, d_{k n}$, and $d_{m n}$ are the distance measurements among the AE source $k$ and sensors $m$ and $n$, respectively. 
In the plane, three adjacent sensors $l, m$, and $n$ are not collinear to avoid the case that $S_{\Delta l m n}=0$. Thus, it remains to determine the sign patterns of the barycentric coordinates for obtaining the barycentric coordinates. In addition, note that $a_{k l}+a_{k m}+a_{k n}=1$. Therefore, given $\left|a_{k l}\right|,\left|a_{k m}\right|$, and $\left|a_{k n}\right|$, the problem of determining the sign of the barycentric coordinate is equated with solving the following equation:

$$
\sigma_{k l}\left|a_{k l}\right|+\sigma_{k m}\left|a_{k m}\right|+\sigma_{k n}\left|a_{k n}\right|=1,
$$

where $\sigma_{k l}, \sigma_{k m}$, and $\sigma_{k n}$ take values of either 1 or -1 . The available information to determine the sign patterns should be limited to the pairwise distance measurements between the AE source $k$ and sensors $l, m$, and $n$.

As shown in Figure 2, there are only 7 possible sign patterns $\left(\sigma_{k l}, \sigma_{k m}\right.$, and $\left.\sigma_{k n}\right)$ of the barycentric coordinates (as the pattern $(-1,-1,-1)$ is not a possible solution for (15)). Therefore, the plane can be divided into 7 zones: I $(1,1,1)$, II $(1,-1,-1)$, III $(1,1,-1)$, IV $(-1,1,-1)$, $\mathrm{V}(-1,1,1)$, VI $(-1,-1,1)$, and VII $(1,-1,1)$. It turns out that sometimes the sign pattern can be uniquely determined from (15), but sometimes this cannot be done. When the sign pattern cannot be uniquely solved from (15), we consider two cases in the following.

In the first case, one of $\left|a_{k l}\right|,\left|a_{k m}\right|$, and $\left|a_{k n}\right|$ equals to zero. That is, the AE source $k$ lies on the line aligned with one of the three edges of the triangle formed by the three adjacent sensors, according to (13). Without loss of generality, say $a_{k l}=0$. For this case, we can take

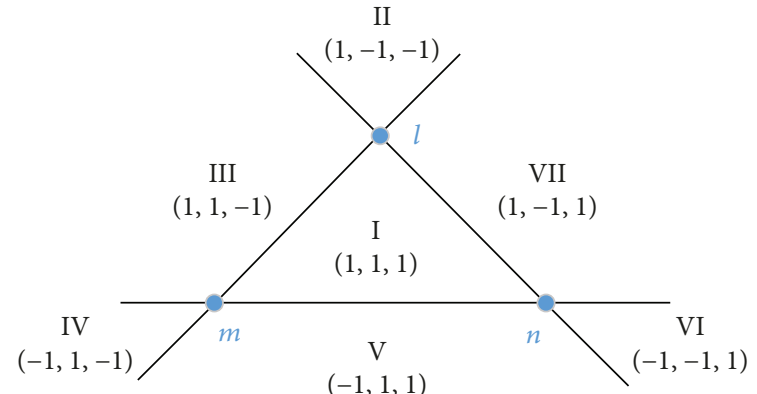

Figure 2: 7 sign patterns for $\left(\sigma_{k l}, \sigma_{k m}\right.$, and $\left.\sigma_{k n}\right)$.

$\sigma_{k l}=1$ (without loss of generality). Then, the other two signs $\sigma_{k m}$ and $\sigma_{k n}$ can be determined according to the following criterion.

$$
\left(\sigma_{k l}, \sigma_{k m}, \sigma_{k n}\right)= \begin{cases}(1,1,1) & \text { if }\left|a_{k m}\right|,\left|a_{k n}\right| \leq 1, \\ (1,1,-1) & \text { if }\left|a_{k m}\right|>1,\left|a_{k m}\right|>\left|a_{k n}\right|, \\ (1,-1,1) & \text { if }\left|a_{k n}\right|>1,\left|a_{k n}\right|>\left|a_{k l}\right| .\end{cases}
$$

In the second case, one of $\left|a_{k l}\right|,\left|a_{k m}\right|$, and $\left|a_{k n}\right|$, saying $a_{k l}$, satisfies $\left|a_{k l}\right|=1$ and $\left|a_{k m}\right|=\left|a_{k n}\right| \neq 0$. For this case, suppose $\angle l m n$ is an acute angle, the sign pattern $\left(\sigma_{k l}, \sigma_{k m}\right.$, and $\left.\sigma_{k n}\right)$ can be determined according to the following criterion.

$$
\left(\sigma_{k l}, \sigma_{k m}, \sigma_{k n}\right)= \begin{cases}(-1,1,1) & \text { if } d_{m k}=d_{l n}, d_{n k}=d_{l m}, d_{l k}^{2}=2 d_{l m}^{2}+2 d_{l n}^{2}-d_{m n}^{2}, \\ (1,1,-1) & \text { if } d_{m k}^{2}<d_{l m}^{2}+d_{l k}^{2}, \\ (1,-1,1) & \text { if } d_{m k}^{2}>d_{l m}^{2}+d_{l k}^{2} .\end{cases}
$$

\section{AE Source Location Experiments}

In this work, all the experiments are performed on a 6061 aluminum alloy (Al-alloy) plate. The dimension of the Al-alloy plate is $500 \mathrm{~mm} \times 500 \mathrm{~mm} \times 2 \mathrm{~mm}$. The diagram of the experimental setup is shown in Figure 3. The data sampling frequency is set at $2 \mathrm{MHz}$. Three FBGs are glued on the preselected points of the Al-alloy plate, whose coordinates are $A=100 \mathrm{~mm}, 200 \mathrm{~mm} ; B=400 \mathrm{~mm}, 200 \mathrm{~mm}$; and $C=250 \mathrm{~mm}, 350 \mathrm{~mm}$. A normalized pencil lead break source (HSU) is used as an AE source.

According to Section 2.3, the location method based on barycentric coordinates needs the distance measurements among $\mathrm{AE}$ source $S$, point $A$, point $B$, and point $C$. The $\mathrm{AE}$ source location geometry is shown in Figure 4. As the distance measurements between three sensors $(A, B$, and $C)$ are known before the experiment, there are three unknown distances, $d_{S A}, d_{S B}$, and $d_{S C}$.

Because of the isotropic characteristic of the Al-alloy plate, the $\mathrm{AE}$ wave travels at the same speed in all directions. As shown in Figure 4, AE wave velocity measurement is carried out at point $P$. Point $P$ is laid on the line $B C$ and the coordinate is $(200,200)$. When there is an $\mathrm{AE}$ event happens at point $P$, the $\mathrm{AE}$ signals obtained by FBGs $(B$ and $C)$ are shown in Figure 5 . The frequency responses of FBG signals are shown in Figure 6. It is known that the $\mathrm{AE}$ signal is a broadband signal with a range from $50 \mathrm{kHz}$ to $300 \mathrm{kHz}$. The Morlet wavelet transform is utilized to extract a narrowband signal with central frequency of $100 \mathrm{kHz}$. As shown in Figure 7, the module 


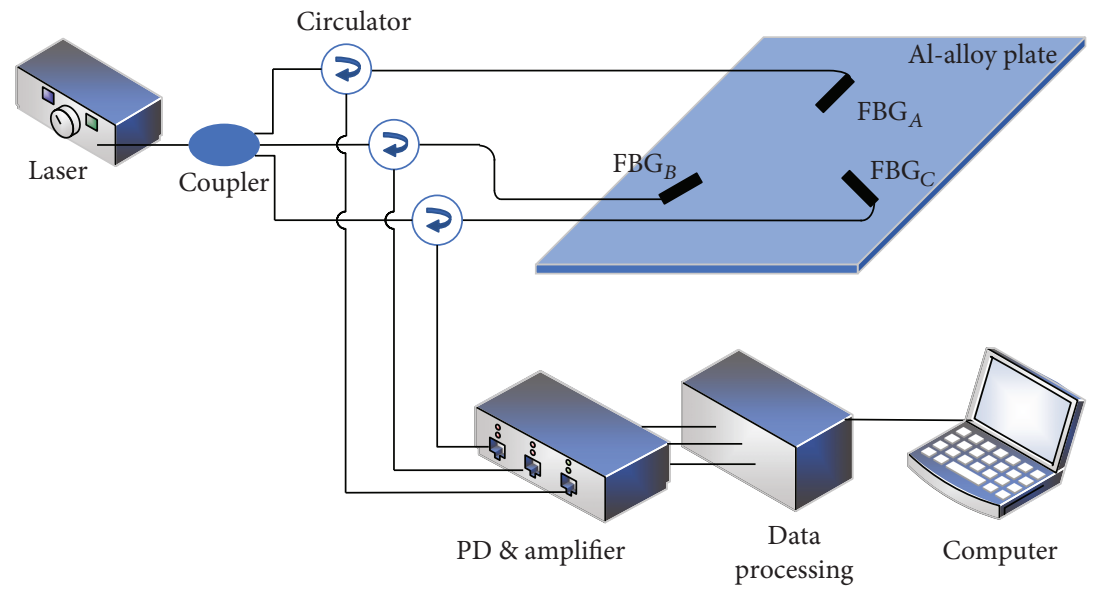

FIgURE 3: The schematic of the experimental setup.

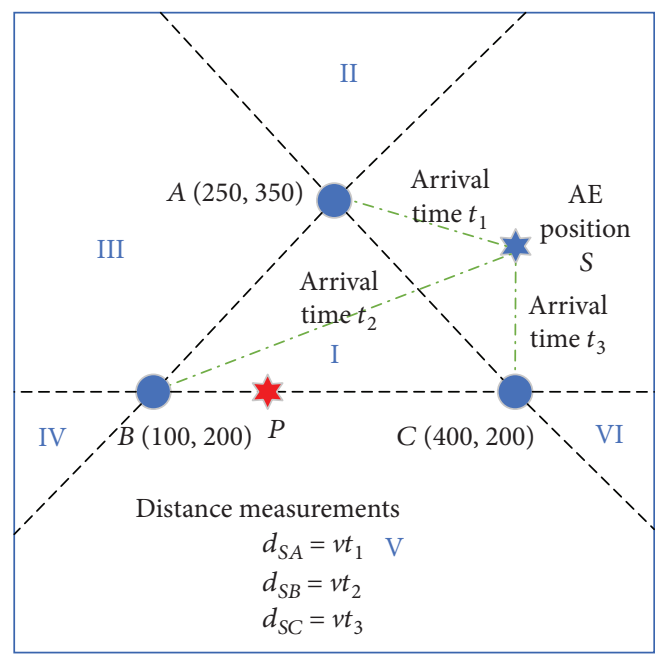

FIgURE 4: The AE source location geometry.

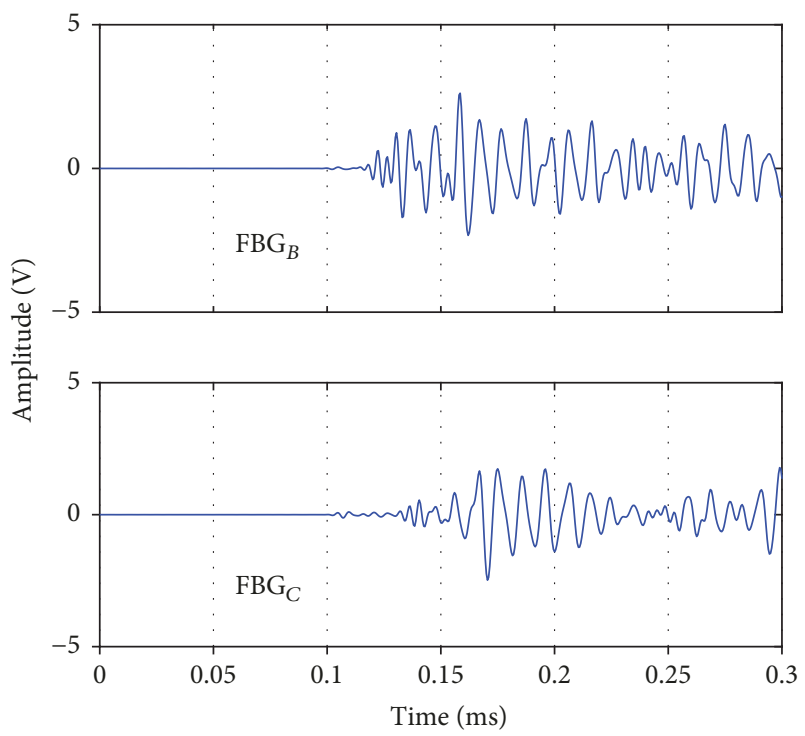

Figure 5: AE waves detected by $\mathrm{FBG}_{B}$ and $\mathrm{FBG}_{C}$.

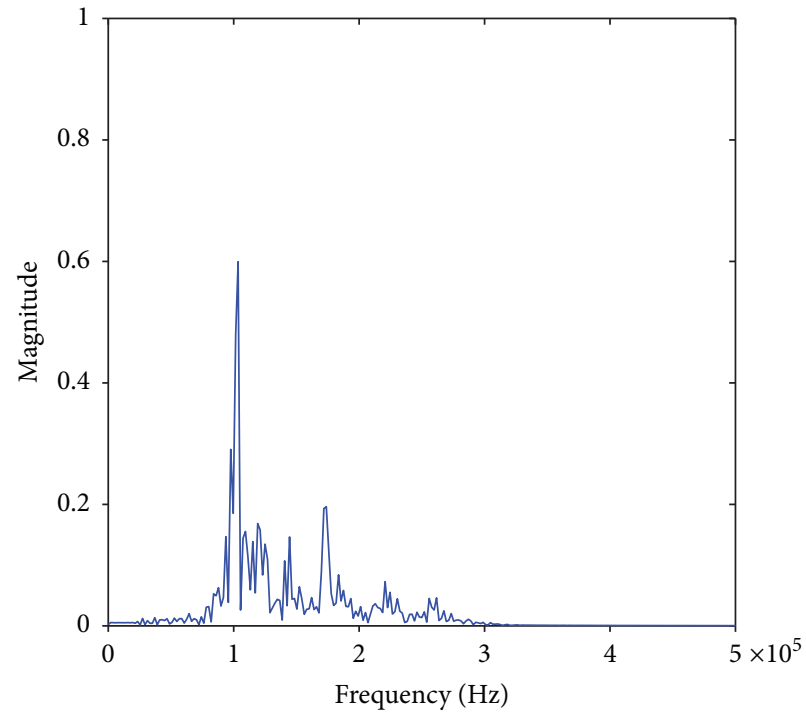

Figure 6: Frequency response of the detected signal.

values of the extracted narrowband signals are obtained and the first peaks of the module values are used to compute the time difference $\Delta t$ between FBGs ( $B$ and $C$ ). Depending on the time difference and the distance difference measured between $P B$ and $P C$, the AE wave velocity $v_{\mathrm{AE}}$ can be obtained. In order to reduce the measurement error, the same experiment is repeated for 10 times and the results are shown in Figure 8. Based on the above method, the average wave velocity is $2454.1 \mathrm{~m} / \mathrm{s}$ and the estimation error is less than $0.25 \%$.

The threshold value method is an effective and simple technique to obtain the arrival times. At the beginning, according to the detected velocity $v_{\mathrm{AE}}$ and the arrival time of the AE wave from point $P$ to point $C, t_{P C}$ is calculated as $t_{P C}=d_{P C} / v_{\mathrm{AE}}$. In Figure 7 , the absolute module value corresponding to $t_{P C}$ is set as the threshold value. In the next experiment, when the module value of the FBG signal is greater than the preset threshold value, this moment is the arrival time of the $\mathrm{AE}$ wave. Based on this process, 


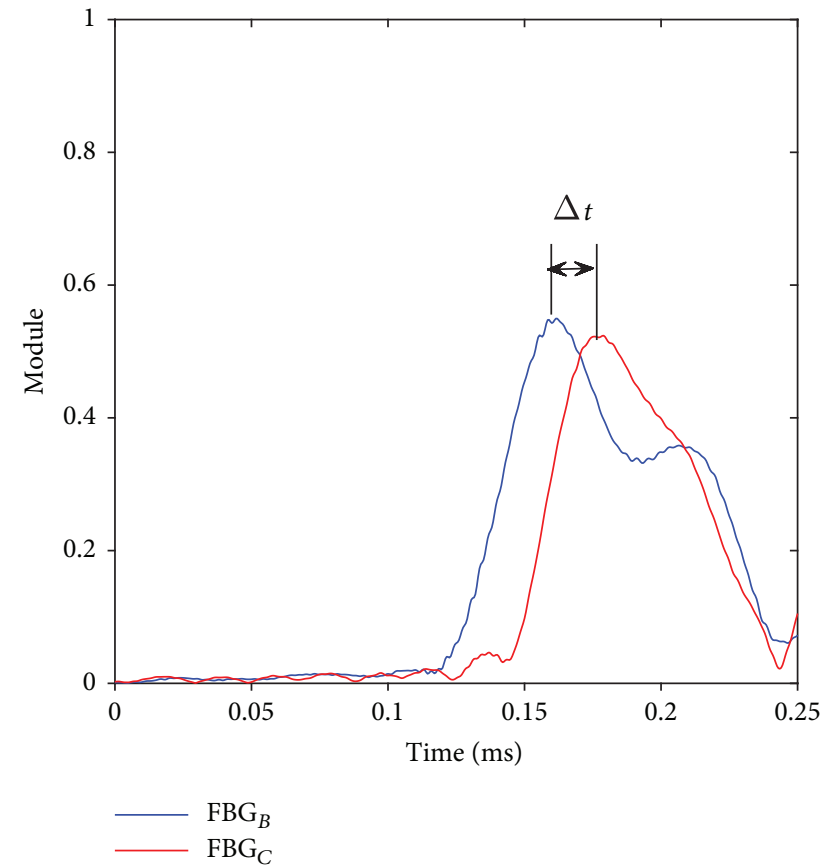

FIGURE 7: Module values of the extracted narrowband signals.

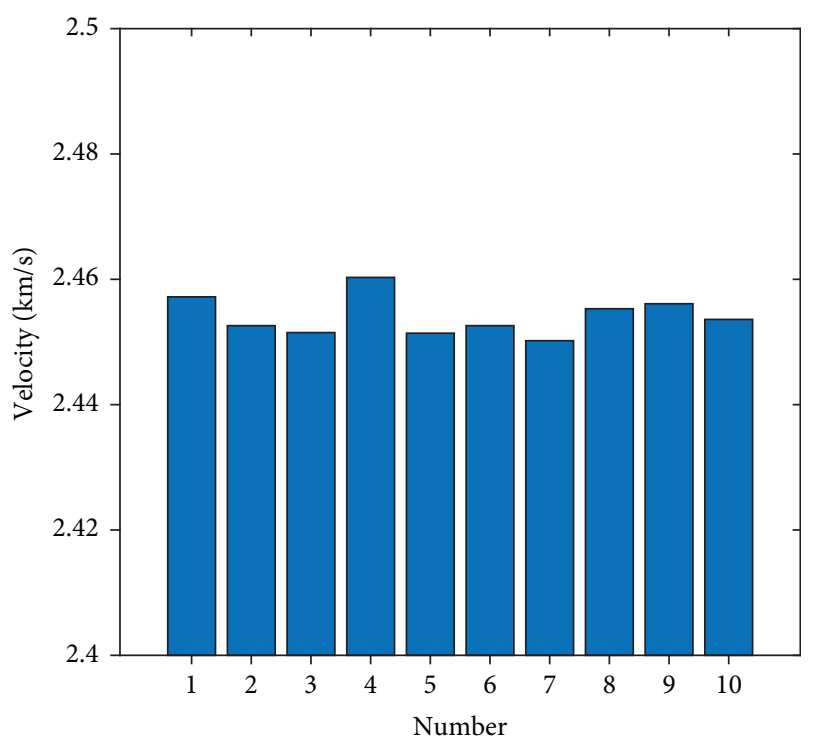

FIgURE 8: Measurement results of wave velocity.

arrival times $\left(t_{1}, t_{2}\right.$, and $\left.t_{3}\right)$ of $\mathrm{AE}$ waves in Figure 4 can be computed.

\section{Results}

Impact is applied on the plate using a steel ball at randomly selected location of $S(400 \mathrm{~mm}, 300 \mathrm{~mm})$, as shown in Figure 4. The AE waves detected by FBGs are shown in Figure 9. After complex Morlet wavelet transform, the narrowband signals are illustrated in Figure 10. According to the narrowband signals, the arrival times of AE signals

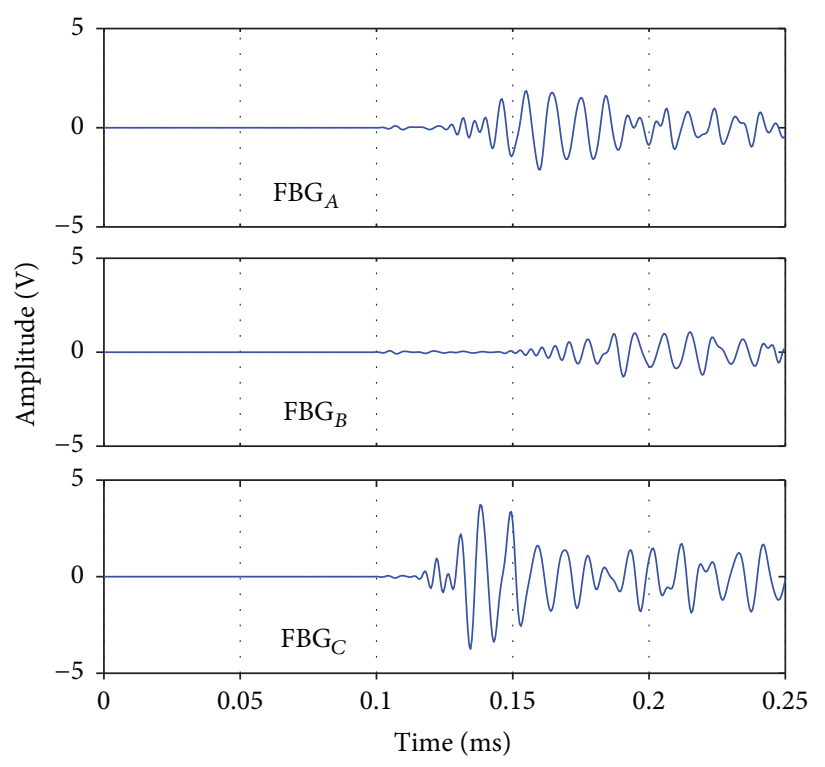

Figure 9: AE waves detected by FBGs.

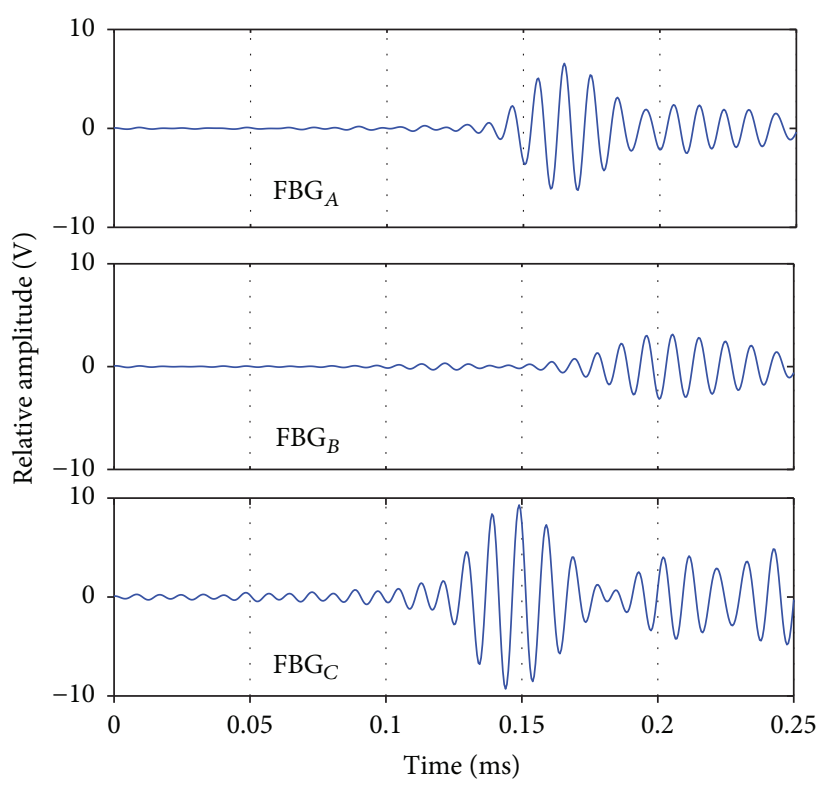

FiguRE 10: Narrowband signals extracted from the detected signals.

are computed. Based on the calculated velocity $v_{\mathrm{AE}}$, the distance measurements among $\mathrm{AE}$ source $S$, point $A$, point $B$, and point $C$ are $d_{A B}=150 \sqrt{2}, d_{A C}=150 \sqrt{2}, d_{B C}=300$, $d_{\mathrm{AS}}=50 \sqrt{10}, d_{B S}=100 \sqrt{10}$, and $d_{C S}=100$, respectively. Then, absolute values of the barycentric coordinates $\left|a_{S A}\right|$, $\left|a_{S B}\right|$, and $\left|a_{S C}\right|$ are calculated. According to (15), (16), and (17), the sign patterns $\left(\sigma_{S A}, \sigma_{S B}\right.$, and $\left.\sigma_{S C}\right)$ can be deduced as $(1,-1,1)$; therefore, the position of impact is correctly found at the zone VII. The Euclidean coordinate $p_{S}$ of the AE source $S$ is calculated.

In each zone, two positions are randomly selected to verify the results of the proposed location method. The location results are presented in Figure 11 and Table 1. 


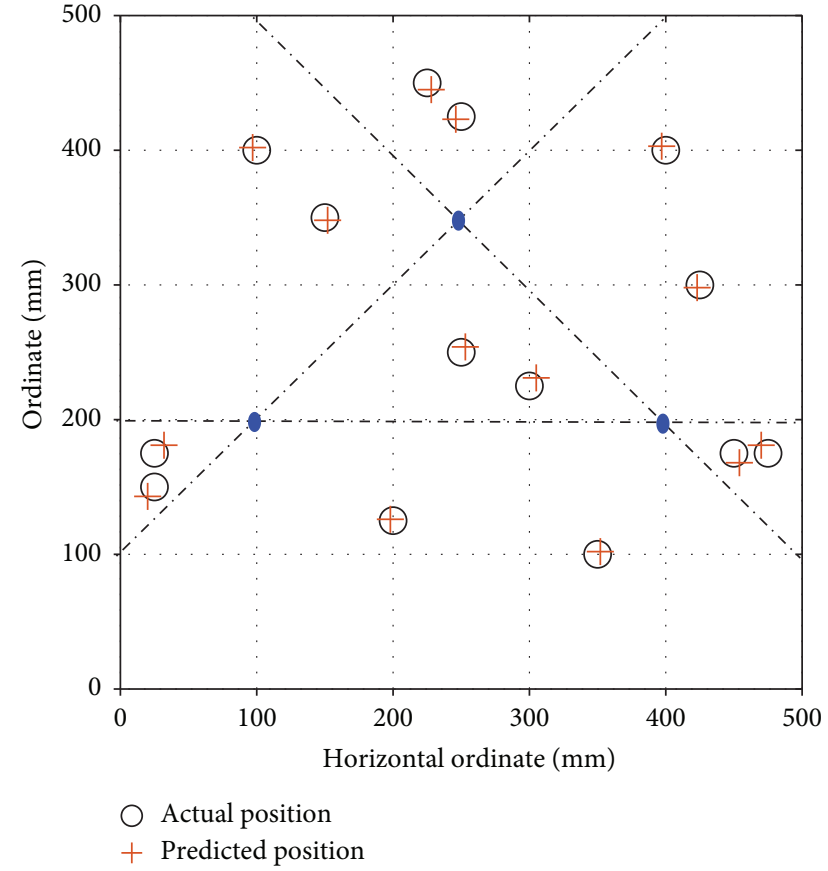

FIgURE 11: The localization results.

TABLE 1: Location results in 7 zones.

\begin{tabular}{lcc}
\hline & Actual position $(\mathrm{mm})$ & Predicted position $(\mathrm{mm})$ \\
\hline $\mathrm{I}$ & $(250,250)$ & $(253,254)$ \\
& $(300,225)$ & $(305,231)$ \\
\hline $\mathrm{II}$ & $(250,425)$ & $(246,423)$ \\
& $(225,450)$ & $(228,445)$ \\
\hline \multirow{2}{*}{ III } & $(150,350)$ & $(152,348)$ \\
& $(100,400)$ & $(97,402)$ \\
\hline \multirow{2}{*}{$\mathrm{IV}$} & $(25,175)$ & $(32,181)$ \\
& $(25,150)$ & $(20,143)$ \\
\hline \multirow{2}{*}{ V } & $(200,125)$ & $(198,126)$ \\
& $(350,100)$ & $(352,102)$ \\
VI & $(450,175)$ & $(454,168)$ \\
& $(475,175)$ & $(470,181)$ \\
\hline \multirow{2}{*}{ VII } & $(425,300)$ & $(423,298)$ \\
& $(400,400)$ & $(397,403)$ \\
\hline
\end{tabular}

The results show that the average and maximum location errors that correspond to the horizontal direction are, respectively, 3.6 and $7 \mathrm{~mm}$. The average and maximum location errors that correspond to the vertical direction are 3.9 and $7 \mathrm{~mm}$, respectively. Also, mean square deviation is employed to calculate the AE source location error using the proposed localization method.

$$
E=\sqrt{\left(x_{\mathrm{p}}-x_{\mathrm{a}}\right)^{2}+\left(y_{\mathrm{p}}-y_{\mathrm{a}}\right)^{2}},
$$

where $\left(x_{\mathrm{p}}, y_{\mathrm{p}}\right)$ is the Euclidean coordinate of the predicted $\mathrm{AE}$ source and $\left(x_{\mathrm{a}}, y_{\mathrm{a}}\right)$ is the Euclidean coordinate of the

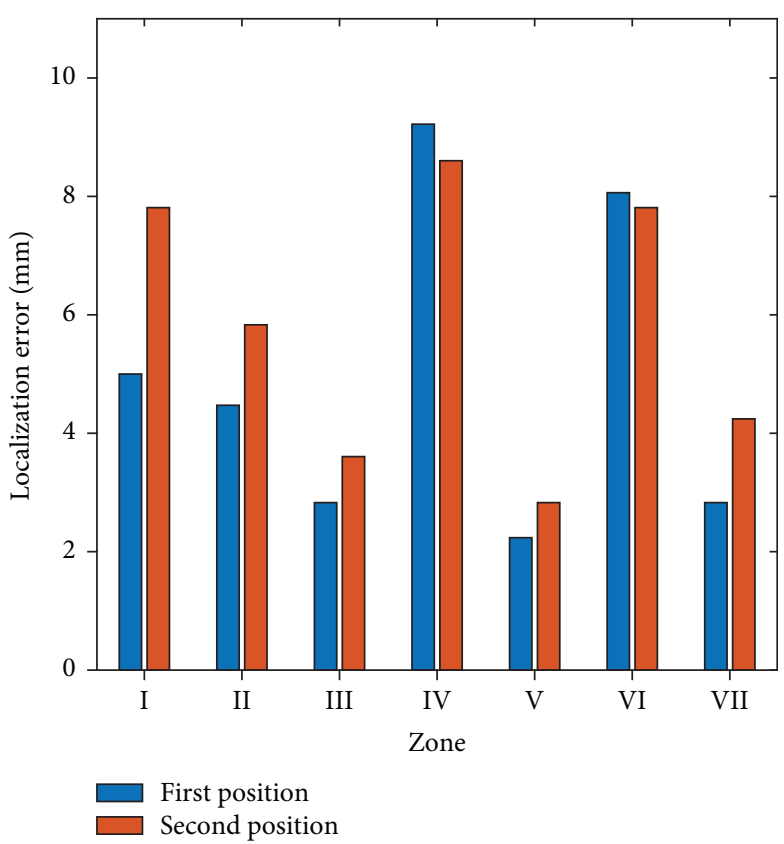

FIgURE 12: The AE source localization errors.

actual AE source. The location errors based on the proposed location method are indicated in Figure 12. The results show that the average and maximum location errors are 5.4 and $9.2 \mathrm{~mm}$, respectively.

\section{Conclusions}

In this paper, we have introduced an easy realizable $\mathrm{AE}$ source localization method based on the $\mathrm{AE}$ waves obtained by FBG sensors. The edge filter method-based FBG interrogation system is used to satisfy the high-speed signal demodulation needs. To realize the barycentric coordinate-based location method, the Morlet wavelet transform is utilized for velocity and arrival time calculation. Then, the AE source location method based on barycentric coordinates is proposed. In the end, impact experiments with random selected positions are carried out for verification of the proposed location method. This research provides an easy and efficient method to safety monitoring of structures.

\section{Data Availability}

The data used to support the findings of this study are available from the corresponding author upon request.

\section{Conflicts of Interest}

The authors declare that they have no conflicts of interest.

\section{Acknowledgments}

This research is supported by the National Natural Science Foundation of China (Grant nos. 61503218, 61773241, 61573226, and 41472260) and the Doctoral Research fund of Shandong Jianzhu University (no. XNBS1423). 


\section{References}

[1] K. Dziedziech, L. Pieczonka, P. Kijanka, and W. J. Staszewski, "Enhanced nonlinear crack-wave interactions for structural damage detection based on guided ultrasonic waves," Structural Control and Health Monitoring, vol. 23, no. 8, pp. 1108-1120, 2016.

[2] T. Stepinski, T. Uhl, and W. Staszewski, Advanced Structural Damage Detection: From Theory to Engineering Applications, John Wiley \& Sons, 2013.

[3] U. Dackermann, Y. Yu, E. Niederleithinger, J. Li, and H. Wiggenhauser, "Condition assessment of foundation piles and utility poles based on guided wave propagation using a network of tactile transducers and support vector machines," Sensors, vol. 17, no. 12, p. 2938, 2017.

[4] T. Druet, B. Chapuis, M. Jules, G. Laffont, and E. Moulin, "Passive SHM system for corrosion detection by guided wave tomography," in Sensors, Algorithms and Applications for Structural Health Monitoring, IIW Collection, B. Chapuis and E. Sjerve, Eds., pp. 21-29, Springer, Cham, 2018.

[5] D. Ozevin, D. W. Greve, I. J. Oppenheim, and S. P. Pessiki, "Resonant capacitive MEMS acoustic emission transducers," Smart Materials and Structures, vol. 15, no. 6, pp. 18631871, 2006.

[6] A. Perelli, L. De Marchi, A. Marzani, and N. Speciale, "Acoustic emission localization in plates with dispersion and reverberations using sparse PZT sensors in passive mode," Smart Materials and Structures, vol. 21, no. 2, article 025010, 2012.

[7] L. De Marchi, N. Testoni, and A. Marzani, "Spiral-shaped piezoelectric sensors for Lamb waves direction of arrival (DoA) estimation," Smart Materials and Structures, vol. 27, no. 4, article 045016, 2018.

[8] Q. Zhang, Y. Zhu, X. Luo, G. Liu, and M. Han, "Acoustic emission sensor system using a chirped fiber-Bragg-grating Fabry-Perot interferometer and smart feedback control," Optics Letters, vol. 42, no. 3, pp. 631-634, 2017.

[9] P. A. Fomitchov and S. Krishnaswamy, "Response of a fiber Bragg grating ultrasonic sensor," Optical Engineering, vol. 42, no. 4, p. 956, 2003.

[10] G. Zhao, S. Li, H. Hu, Y. Zhong, and K. Li, "Impact localization on composite laminates using fiber Bragg grating sensors and a novel technique based on strain amplitude," Optical Fiber Technology, vol. 40, pp. 172-179, 2018.

[11] M. Kurohiji, S. Ichiriyama, N. Yamasaku et al., "A robust fiber Bragg grating hydrogen gas sensor using platinum-supported silica catalyst film," Journal of Sensors, vol. 2018, Article ID 5810985, 8 pages, 2018.

[12] T. Fu, Z. Zhang, Y. Liu, and J. Leng, "Development of an artificial neural network for source localization using a fiber optic acoustic emission sensor array," Structural Health Monitoring, vol. 14, no. 2, pp. 168-177, 2015.

[13] M. Jiang, S. Lu, Y. Sai, Q. Sui, and L. Jia, "Acoustic emission source localization technique based on least squares support vector machine by using FBG sensors," Journal of Modern Optics, vol. 61, no. 20, pp. 1634-1640, 2014.

[14] F. Yu, Y. Okabe, Q. Wu, and N. Shigeta, "Fiber-optic sensor-based remote acoustic emission measurement of composites," Smart Materials and Structures, vol. 25, no. 10, article 105033, 2016.

[15] J. Yu, F. Xu, and X. Bingsheng, "Acoustic emission tomography based on simultaneous algebraic reconstruction technique to visualize the damage source location in Q235B steel plate," Mechanical Systems and Signal Processing, vol. 64-65, pp. 452-464, 2015.

[16] Y. Sai, X. Zhao, D. Hou, and M. Jiang, "Acoustic emission localization based on FBG sensing network and SVR algorithm," Photonic Sensors, vol. 7, no. 1, pp. 48-54, 2017.

[17] C. Li, Z. Ding, D. Zhao, J. Yi, and G. Zhang, "Building energy consumption prediction: an extreme deep learning approach," Energies, vol. 10, no. 10, p. 1525, 2017.

[18] X. Cheng, X. Zhang, L. Zhao et al., "The application of Shuffled Frog Leaping Algorithm to Wavelet Neural Networks for acoustic emission source location," Comptes Rendus Mecanique, vol. 342, no. 4, pp. 229-233, 2014.

[19] H. Sadegh, A. N. Mehdi, and A. Mehdi, "Classification of acoustic emission signals generated from journal bearing at different lubrication conditions based on wavelet analysis in combination with artificial neural network and genetic algorithm," Tribology International, vol. 95, pp. 426-434, 2016.

[20] X. Yu, Y. Yu, M. Zhang, Y. Liao, and S. Lai, "Study on the strain and temperature sensing characteristics of FBG packaged by the copper slice," Acta Photonica Sinica, vol. 35, no. 9, pp. 1325-1328, 2006.

[21] S. Tao, X. Zhou, and Z. Zhang, "On fault feature extraction of a gear by complex Morlet wavelet transform and coefficient correlation," Mechanical Science and Technology for Aerospace Engineering, vol. 5, p. 19, 2010.

[22] N. G. Nikolaou and I. A. Antoniadis, "Demodulation of vibration signals generated by defects in rolling element bearings using complex shifted Morlet wavelets," Mechanical Systems and Signal Processing, vol. 16, no. 4, pp. 677-694, 2002.

[23] H. Coxeter, Introduction to Geometry, Wiley, New York, NY, USA, 1969.

[24] Y. Diao, Z. Lin, and M. Fu, "A barycentric coordinate based distributed localization algorithm for sensor networks," IEEE Transactions on Signal Processing, vol. 62, no. 18, pp. 47604771, 2014.

[25] J. Warren, S. Schaefer, A. N. Hirani, and M. Desbrun, "Barycentric coordinates for convex sets," Advances in Computational Mathematics, vol. 27, no. 3, pp. 319-338, 2007.

[26] T. Han, Z. Lin, R. Zheng, and M. Fu, "A barycentric coordinate-based approach to formation control under directed and switching sensing graphs," IEEE transactions on cybernetics, vol. 48, no. 4, pp. 1202-1215, 2018. 


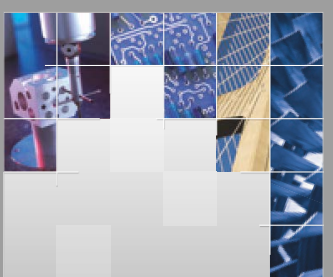

\section{Enfincering}
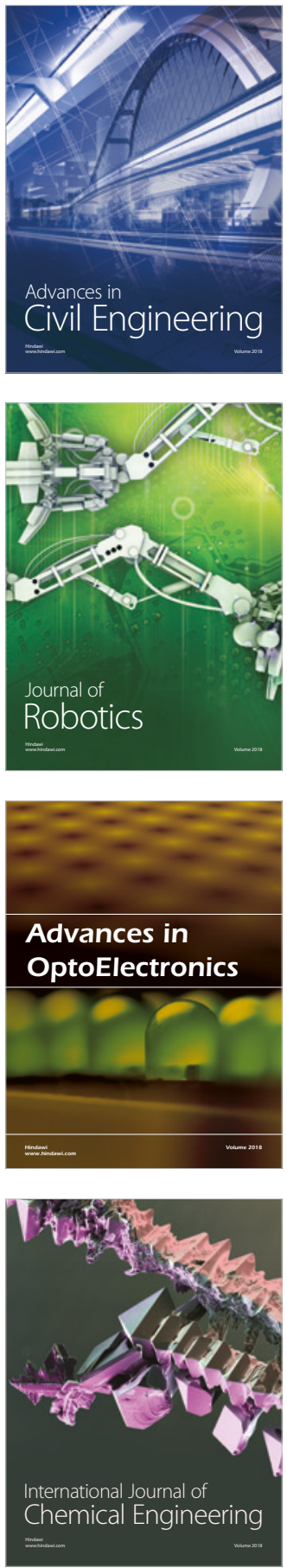

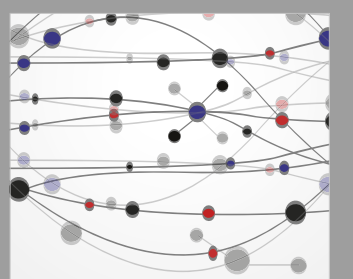

\section{Rotating \\ Machinery}

The Scientific World Journal

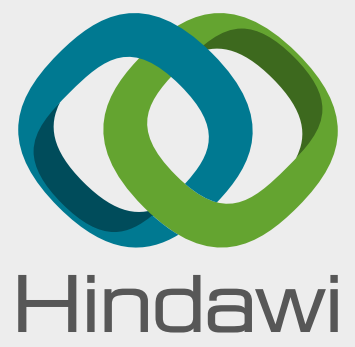

Submit your manuscripts at

www.hindawi.com
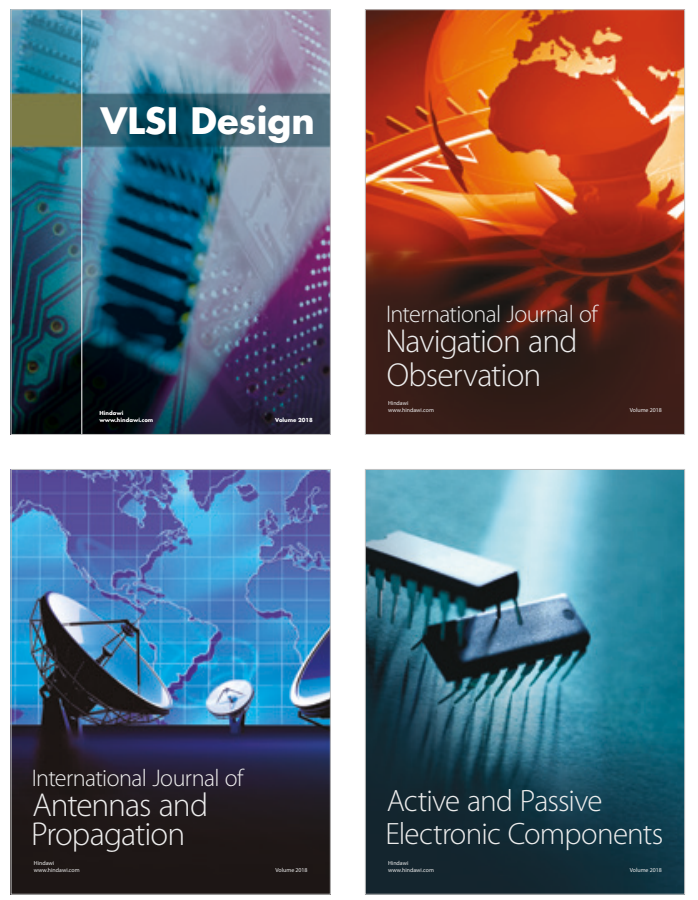
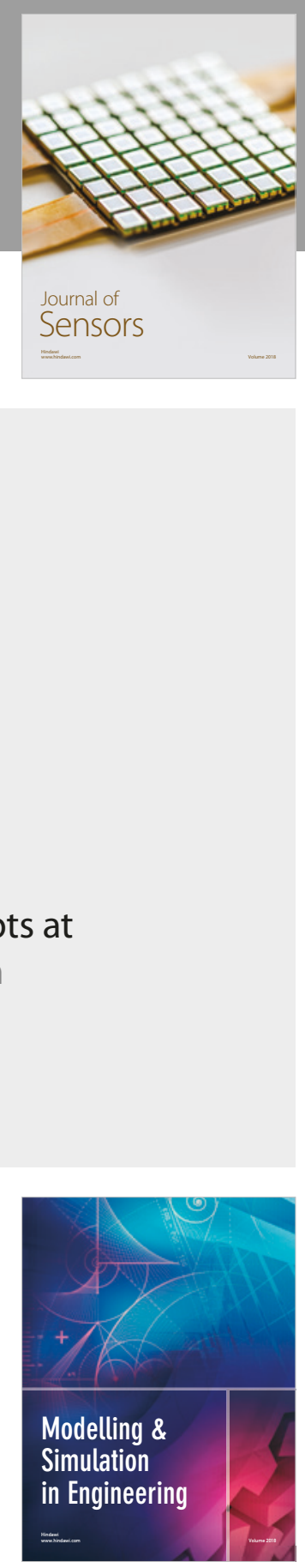

\section{Advances \\ Multimedia}
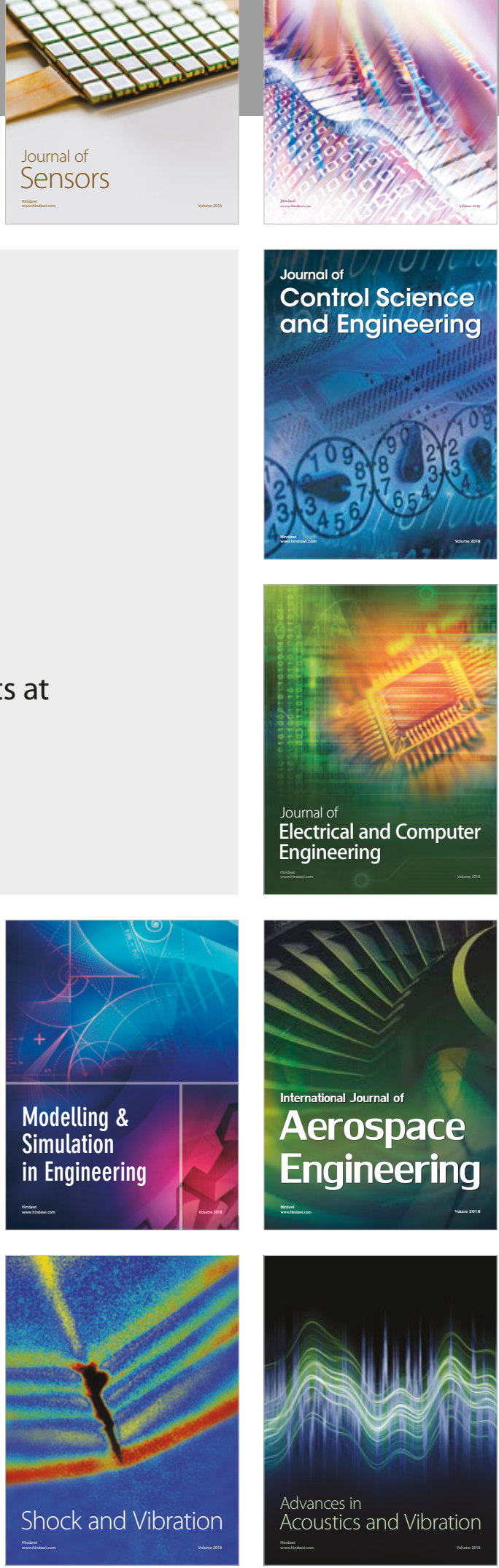